\title{
Integrated physical enhanced recovery method for high-viscosity oil reservoirs
}

\author{
Aziz Khudaiberdiev ${ }^{1, *}$, and Petr Kosianov ${ }^{1}$ \\ ${ }^{1}$ Industrial University of Tyumen, Nizhnevartovsk, Lenina str. 2P, building 9, Nizhnevartovsk, 628600, \\ Russia
}

\begin{abstract}
The physical methods of enhanced oil recovery using electromagnetic fields are studied in this paper. Purpose of the work is to study the dependence of the main quantities that determine the volume of filtered oil, including the viscosity of oil, on the parameters (temperature, intensity and frequency) of thermal and electromagnetic fields, and optimize these parameters for maximum oil recovery factor using electric fields and steam treatment of the formation.It is proposed to combine the most effective and environmentally friendly methods to increase oil production. In the developed technique, methods of converting steam energy are used to create a torque of the drilling device with simultaneous steam treatment of the bottomhole zone of the reservoir. As well as the impact of an alternating electromagnetic field on the reservoir matrix and interstratal liquid fluids to create currents, increase the mobility of molecules of liquid fluids, and, as a consequence, increase the temperature and lower the viscosity of oil, which will increase oil recovery. As a result of numerous experimental experiments carried out using the original setup in the laboratory of the branch of the Tyumen Industrial University in Nizhnevartovsk, it was shown that a decrease in viscosity is observed only when exposed to simultaneous thermal and electromagnetic fields.
\end{abstract}

\section{Introduction}

The object of the study is to increase the oil recovery factor of the reservoir.

Subject - physical methods of increasing the oil recovery factor using electromagnetic fields.

The tasks that need to be solved to achieve the goal:

1. Conduct a detailed analysis of the application of existing physical methods.

2. Study the optimal parameters of the thermal-steam treatment method.

3. Study the optimal parameters of the method using electric fields.

4. Integrate the method of thermal-steam exposure and method of electric fields to develop a new way for increasing oil recovery.

According to expert estimates, residual oil reserves (100\%) are quantitatively distributed by types as follows:

1. oil remaining in low-permeability inter-layers and areas not covered by water $-27 \%$;

2. oil in stagnant zones of homogeneous formations $-19 \%$;

\footnotetext{
*Corresponding author: aziz.5199@mail.ru
} 
3. oil remaining in the lenses and near impermeable screens not penetrated by wells $-24 \%$;

4. capillary-retained and film oil - $30 \%$

Residual oil (items 1-3), which is not covered by the waterflooding process due to the high macro-heterogeneity of the developed reservoirs and stagnant zones formed by fluid flows in the reservoirs, make up $70 \%$ of all residual reserves, representing the main reserve for increasing oil recovery. [1] It is possible to increase the oil recovery of the reservoir due to this part of the oil as a result of improving the existing systems and technologies for development and the so-called hydrodynamic methods of increasing oil recovery. The rest (item 4) remains in the flooded reservoirs due to their microheterogeneity and can be recovered only as a result of the impact of various physical and physicochemical processes and phenomena. Every year, 8-10 thousand treatments of injection wells with a specific technological effect of 1.4-1.5 thousand tons of additional oil per operation are carried out at Russian oilfields. In recent years, many oil companies have focused on the use of such technologies as hydraulic fracturing, forced fluid withdrawal (FFW), sidetracking, and horizontal drilling. There is no doubt that these technologies have high technical and economic efficiency. However, in the conditions of highly watered reservoirs, which are at a late stage of development and are characterized by a high degree of reserve recovery, where the effective use of these methods is limited, flow control technologies for influencing the injection well stock allow obtaining high results: increasing the oil recovery factor (ORF) by $2-15 \%$ and reducing the cost of oil production by $1.2-2$ times.

During the research, we studied various works on enhancing oil recovery by various methods, some of which are described in the work. [2-7] Existing methods, having a high oil recovery rate, also have the disadvantage of aggressive environmental impact. [8] Therefore, we will focus on more environmentally friendly ones - physical methods that are not inferior in terms of oil recovery. Physical methods have great development prospects and are environmentally friendly.

We conducted a comparative analysis of existing patents for inventions in order to find out their main advantages and disadvantages:

Method for the development of oil and gas condensate fields. According to this method, an emitter of electromagnetic waves and, together with or separately, an electrode of the high frequency electric field are placed in the well.

In the first two wells, a decrease in viscosity by $32 \%$ was observed. In the second two wells, a decrease in viscosity by $15-20 \%$ occurred. In a well with a high paraffin content, the viscosity decreased by $15 \%$, and the decrease in wax percentage was $15 \%$. According to the results of the check, the flow rate of fluid in the wells before treatment using the proposed technology was 52 tons/day, and after - 89 tons/day, i.e. increased by 27 tons/day (58\%). [9]

An unconventional drilling method. The work of steam energy is performed both due to its exit from the nozzles installed on the working body, and due to the action of the jet on the walls of the well.

By generating steam energy inside the body of the drilling tool and using this energy through the outlet channels of the body, mechanical work is performed. This eliminates the loss for steam transportation. [10]

Electrochemical method of enhanced oil recovery. The method is based on an additional action of a direct electric current on the reservoir. This current is passed within the well cluster through two supply electrodes located at the reservoir level. [11]

A preliminary comparative analysis showed that it is the physical methods of increasing the oil recovery factor $(\mathrm{ORF})$ that are more environmentally friendly compared to chemical methods, which involve the injection of chemicals into the reservoir. In fact, the interstratal 
fluids of the fields being developed already contain alkalis and acids, which are additionally injected into the reservoir using chemical methods.

\section{Materials and methods}

The developed method will use the methods of converting steam energy to create the torque of the drilling device with simultaneous thermal-steam effect on the bottom-hole zone of the reservoir and the effect of an electrostatic field on the reservoir matrix and interstratal liquid fluids to create currents, which will increase oil recovery.

To assess the efficiency of the device under thermal steam exposure, its optimal parameters were determined.

Thermal and electromagnetic fields, interacting with reservoir fluids, lead to the appearance of additional pressure gradients, promote additional recovery of film and capillary bound oil, which leads to an increase in the displacement efficiency. The energy imparted by the electric fields is used to destroy the bonds of the spatial structure of oil, which directly increases the permeability of the reservoir, and accordingly enhances oil recovery.

Formulation of the problem. Estimation of device parameters.

In this paper, it is proposed to integrate methods for converting steam energy to create a drilling device torque, steam-thermal impact on the bottom-hole reservoir zone, and the effect of an electrostatic field on the reservoir matrix and interstratal liquid fluids to create currents and enhance oil recovery [12]. [13]

To evaluate the efficiency of the device, the parameters of the torque were determined.

In [13], the pressure $p$ and temperature $T$ of steam were determined during the evaporation of a given mass of water, but to calculate the reactive forces $F_{r}$ and the torque $M_{f}$, it is necessary to first determine the rate of steam outflow from the nozzles of the device (see figure 1).

With the evaporation of $1 \mathrm{~kg}$ of water for 1 second, inside the cavity of the device with a volume of $V=10^{-3} \mathrm{~m}^{3}$, the pressure of the superheated steam in front of the nozzle is $p_{I}=$ $460 * 10^{5} \mathrm{~Pa}$, the temperature is $\mathrm{T}=998 \mathrm{~K}$.

The pressure behind the nozzle $p_{2}=10^{5} \mathrm{~Pa}$. The ratio of pressures in the environment and in front of the nozzle $\beta=p_{2} / p_{1}=2,17 * 10^{-3}$.

The critical value of the ratio (with sound steam flow) is determined by the expression [14]:

$$
\beta_{c r}=\left(\frac{2}{k+1}\right)^{\frac{k}{k-1}}
$$

where $k$-adiabatic exponent of the flow, for superheated steam $k=1.29$, so $\beta_{c r}=0.547$. Since $\beta<<\beta_{c r}$, the outflow is supersonic. 

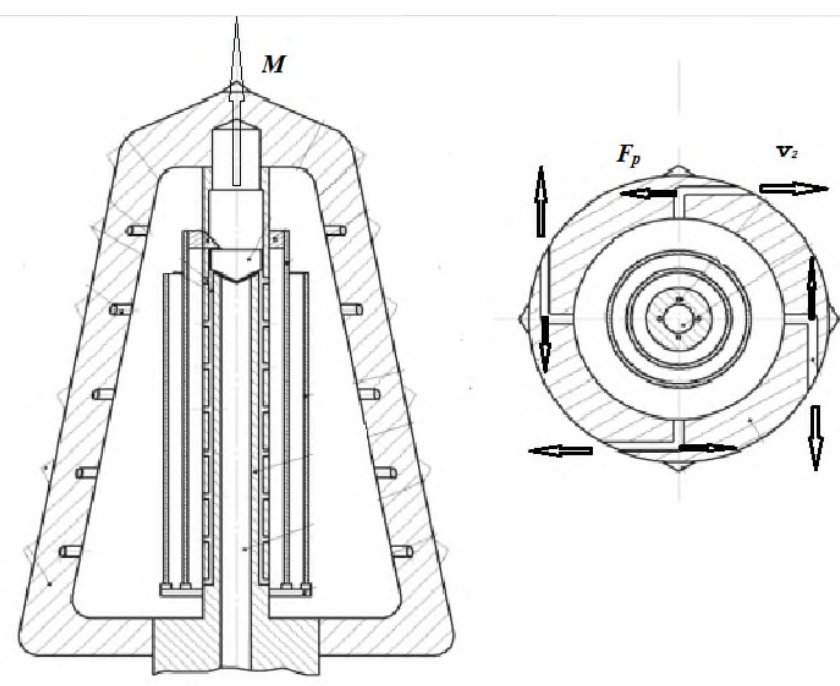

Fig. 1. Steam outflow from nozzles with speed $\boldsymbol{v}_{2}$, arising reactive forces $\boldsymbol{F}_{\boldsymbol{r}}$ and torque (moment of forces) $\boldsymbol{M}$ acting on the device

The velocity at the exit from the nozzle for a real gas is determined by the expression:

$$
v_{2}=\sqrt{2}\left(i_{1}-i_{2}\right)
$$

where: $i_{1}$ - enthalpy of steam at the nozzle inlet; $i_{2}$ - enthalpy of steam at the exit from the nozzle.

Enthalpy is found by is - diagram [15].

For the problem being solved: $i_{1}=3785 \mathrm{KJ} / \mathrm{kg} ; i_{2}=2935 \mathrm{KJ} / \mathrm{kg}$.

The velocity at the exit from the nozzle $v_{2}=1304 \mathrm{~m} / \mathrm{s}$. With an expanding shape of the nozzle with an aperture angle $\gamma \approx 10^{\circ}$ (see figure 3), the velocity coefficient of the nozzle is $\operatorname{maximum} \varphi=0.98$ and the actual outflow velocity is $v_{2}{ }^{a}=\varphi v_{2}=1278 \mathrm{~m} / \mathrm{s}$.

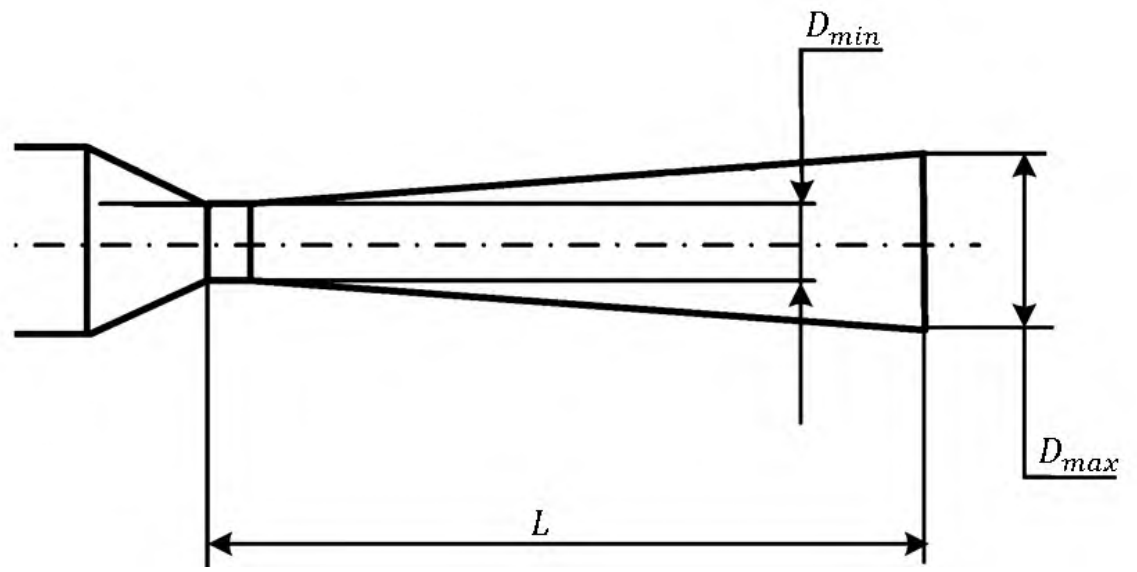

Fig. 2. Nozzle with an expanding shape, $D_{\min }$ - minimum nozzle diameter, $D_{\max }$ - maximum nozzle diameter, L - nozzle length. 
The nozzle parameters are related by the following expression:

$$
\tan \left(\frac{\gamma}{2}\right)=\frac{\text { Dmax }- \text { Dmin }}{2 L}
$$

Reactive force $F r=v 2 \mathrm{a} \cdot \mu$, where $\mu=\frac{d m}{d t}$ is the mass of steam ejected from one nozzle per unit time. In the problem being solved, $\mu=0.25 \mathrm{~kg} / \mathrm{s}$ and $F_{r}=319.5 \mathrm{~N}$. Assuming that the nozzles are identical, the resulting force is $F=4 F_{r}=1278 \mathrm{~N}$. Torque (moment of force) $M_{f}=\mathrm{F} d=4 F_{r} d$, here $d=10 \mathrm{~cm}$ is the shoulder of the force action. Accordingly, $M_{f}=$ $127.8 \mathrm{~N} * \mathrm{~m}$. It is clear that the reactive forces and torque can be adjusted by changing the amount of water supplied to the device (Table 1).

Table 1. Change of parameters depending on the amount of supplied water

\begin{tabular}{|c|c|c|c|c|c|}
\hline $\mathrm{m}(\mathrm{w}), \mathrm{kg}$ & 1 & 2 & 3 & 4 & 5 \\
\hline$\frac{d m}{d t}, \mathrm{~kg}$ & 0.25 & 0.5 & 0.75 & 1.0 & 1.25 \\
\hline $4 F_{r,} \mathrm{~N}^{*} \mathrm{~m}$ & 1278 & 2556 & 3834 & 5112 & 6390 \\
\hline$M_{f}$ & 127.8 & 255.6 & 383.4 & 511.2 & 639.0 \\
\hline $\begin{array}{c}\mathrm{S}_{\text {efficiency }} \\
\mathrm{m}^{2}\end{array}$ & 0.04 & 0.08 & 0.12 & 0.16 & 0.20 \\
\hline
\end{tabular}

Based on the data in the table, we can say that the efficiency (evaporation rate) of water evaporation can be achieved by using water (mist) injection onto the heating element, the surface of which is maximized by corrugation.

In the electrochemical method, chemical reagents are additionally pumped to increase currents in liquid fluids.

Let's consider the electrical properties of aqueous solutions in natural conditions. The most common solutions of salts are $\mathrm{NaCl}, \mathrm{KCl}, \mathrm{MgCl} 2, \mathrm{Na} 2 \mathrm{SO} 4, \mathrm{CaCl} 2, \mathrm{NaHCO} 3$. The resistivity of these solutions of a binary monovalent electrolyte is written by the expression

$$
\rho_{w .20}=\frac{10}{(U+v) C_{w}}=\frac{10}{\wedge C_{w}}[\mathrm{Ohm} * \mathrm{~m}]
$$

where $\mathrm{U}$ and $v$ - cation and anion mobility; $\wedge$ - equivalent electrical conductivity of the electrolyte at $\mathrm{T}=\left(20^{\circ} \mathrm{C} \mathrm{Ohm}-1\right)^{*} \mathrm{~cm} 2, \mathrm{Cw}$ - electrolyte concentration g-eq/1.

With increasing temperature, the resistivity will change as follows:

$$
\rho_{w \mathrm{~T}}=P_{T} \rho_{w 20}=\frac{\rho_{w 20}}{1+\alpha_{T}\left(T-20^{\circ} \mathrm{C}\right)^{\prime}}
$$

where $-\alpha_{\mathrm{T}}-$ temperature coefficient of conductivity; 


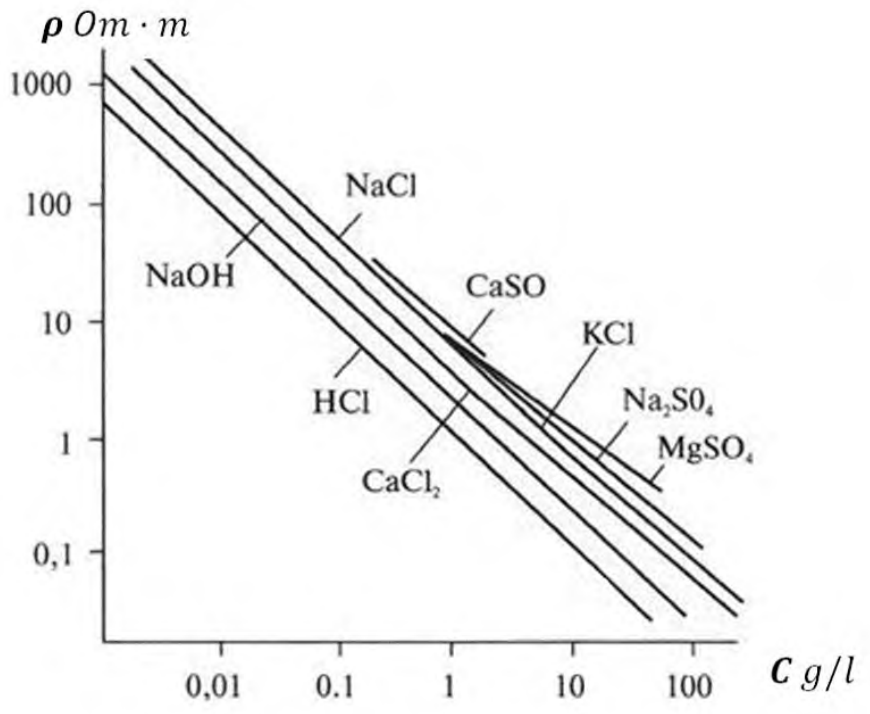

Fig. 3. The dependence of the electrical resistivity on the concentration

In the case of a multicomponent composition, $\rho_{w}$ is calculated by the formula

$$
\rho_{w}=\frac{10}{\sum_{i=1}^{n} \wedge_{i} C_{i}}
$$

where $\Lambda_{i}$ and $C_{i}$ - equivalent electrical conductivity and concentration of the $i$-th electrolyte in a solution containing $n$ electrolytes. [16]

As can be seen from figure 3, the resistivity decreases, and the currents increase, due to which additional heat is released, which accordingly leads to a decrease in the viscosity coefficient.

One of the main problems in the study of the properties of oil is the expression of the viscosity of oil in an explicit form, which is a large and urgent scientific task. To date, the viscosity is determined in the laboratory by the filtered volumes. In the model under consideration, an increase in oil production implies an increase in reservoir pressure, a decrease in viscosity and permeability, and the influence of external physical factors. Modern geophysics uses all kinds of physical fields. One of the most environmentally friendly ways to develop:

Steam-thermal effect on the formation, $\mathrm{K}<70 \%$

Electromagnetic, $\mathrm{K} \leq 70 \%$

The dielectric permittivity of gases is almost independent of temperature. Accordingly, the current density is

$$
\vec{\jmath}=\left(\sigma+\omega \varepsilon^{\prime \prime}+i \omega \varepsilon^{\prime}\right) \vec{E}
$$

To determine the current density, we used the concentration range from minimum to maximum and obtained the following data: the concentration of oil varies from 0.2 to 0.15 , water from 0.5 to 0.8 , gas from 0.3 to 0.05 .

We take the resistivity of oil as $10^{12} \mathrm{Ohm} * \mathrm{~m}$, water $10^{2} \mathrm{Ohm} * \mathrm{~m}$ and gas $10^{14} \mathrm{Ohm} * \mathrm{~m}$. 


$$
\sigma_{l . f .}=\sum_{i=1}^{n} \wedge_{i} c_{i}=\sum \frac{c_{i}}{p_{i}}
$$

Where $c_{i}$ - concentration, $\mathrm{p}$ - resistivity, $\sigma$ - conductivity.

First, let's calculate the sum of the electrical conductivity of these substances

$$
\sigma=\frac{\mathrm{c}_{w}}{\mathrm{p}_{w}}+\frac{\mathrm{c}_{o}}{\mathrm{p}_{o}}+\frac{\mathrm{c}_{g}}{\mathrm{p}_{g}}+\frac{\mathrm{c}_{S}}{\mathrm{p}_{s}}
$$

Because the specific conductivity of oil and gas is very small, we neglect it.

To determine the average conductivity of salt solutions, we use these maximum values of concentration and resistivity of salt fluids that make up oil (Table 2).

Table 2. Maximum values of concentrations and resistivity of salt fluids

\begin{tabular}{|c|c|c|}
\hline Salt solutions & Concentration, $c_{i}$ & $\begin{array}{c}\text { Electrical conductivity, } \\
\Lambda_{i}\end{array}$ \\
\hline $\mathrm{KCl}$ & 0.003 & $4.5 * 10^{-3}$ \\
\hline $\mathrm{Na}_{2} \mathrm{CO}_{3}$ & 0.0017 & $16 * 10^{-3}$ \\
\hline $\mathrm{NaCl}$ & 0.0026 & $83 * 10^{-3}$ \\
\hline $\mathrm{NaOH}$ & 0.0026 & $32 * 10^{-3}$ \\
\hline $\mathrm{BaCO}_{3}$ & 0.0028 & $2.5 * 10^{-3}$ \\
\hline
\end{tabular}

Values calculated according to the table for salt solutions:

$$
\mathrm{p}_{s}=\frac{10}{\sum_{i=1}^{n} \wedge_{i} c_{i}} \approx 25.3 \mathrm{Ohm} \times \mathrm{m}
$$

Thus:

$$
\sigma_{s}=\frac{1}{\mathrm{p}_{s}} \approx 39.5 * 10^{-3} \mathrm{Sim} / \mathrm{m}
$$

Using the given values of the electric field strength

$$
\mathrm{E}=\frac{U_{m}}{d}=\frac{8}{0.28}=28.6 \mathrm{~V} / \mathrm{m},
$$

Where $U_{m}$ - peak voltage, $\mathrm{d}$ - distance between plates of a dismountable capacitor.

Next, we calculate the current density for a liquid fluid:

$$
J=\sigma E \approx 1.1 \frac{\mathrm{A}}{\mathrm{m}^{2}}
$$

\section{Results and discussion}

Let's consider to what depth electromagnetic waves from exposure can penetrate.

Depth of penetration of electromagnetic waves:

$$
R=\sqrt{2 \frac{2}{\omega \gamma \mu \mu_{0}}}
$$

where $\mathrm{R}$-depth of penetration of electromagnetic waves into the rock $(\mathrm{m})$; $\omega$ - cyclic frequency of electromagnetic oscillations $(\mathrm{Hz})$; 
$\mu$ - here - the magnetic permeability of the medium $(\mathrm{H} / \mathrm{m})$;

$\gamma$ - specific electrical conductivity of the medium $[(\mathrm{Ohm} * \mathrm{~m})-1]$

Cyclic frequency:

$$
\omega=2 \pi f
$$

where $f$ - frequency of electromagnetic waves $(\mathrm{Hz})$.

$$
R=\sqrt{\frac{2}{2 * 3.14 * 1094 * 10^{3} \mathrm{~Hz} * 1 * 1.257 * 10^{-6} * 1 * 10^{-6}}}=482(\mathrm{~m})
$$

\section{Conclusions}

The effect of an electromagnetic field on an oil flow reduces the intensity of surface waxing, since an additional charge arises on a charged particle in the flow due to electromagnetic induction. A force will act on the particle from the side of the field, deflecting it towards the region of charges and interaction of charged particles in the flow.

The electromagnetic field, interacting with reservoir fluids, leads to the appearance of additional pressure gradients $\Delta \mathrm{P}$. These phenomena arise due to the appearance of oscillations of hydrocarbon molecules in the region of a high-frequency electromagnetic field as a result of an increase in the mobility of molecules and an increase in temperature. This facilitates additional recovery of film and capillary-bound oil, which leads to an increase in the displacement efficiency. The results of experimental studies have shown a significant increase in oil recovery. According to the obtained results, we can say that with an increase in temperature under laboratory conditions, the viscosity of oil decreases several times, which, accordingly, doubles the volume of filtered oil (by 100\%).

An important difference between the method of exposure to electromagnetic fields and many other methods is their environmental safety, both for the subsoil and the environment.

\section{References}

1. N. V. Korshunov, The effectiveness of methods to increase on the basis of laboratory and experimental studies Modern Innovation, 6, 19-21 (2019)

2. A. V. Afanasyev, Electrochemical method of enhanced oil recovery with artificial waterflooding VI International Youth Scientific Conference "Svergeoecotech-2015" ed. K V Rochev, 191 - 194 (Ukhta: Ukhta State Technical University, 2015)

3. O. Yu. Morozova, O. Yu. Bondarev, Application of downhole electric steam generatorseparator for steam-heat treatment of high-viscosity oil reservoirs VI International Youth Scientific Conference "Svergeoecotech-2015” ed. K V Rochev, 252 - 256 (Ukhta: Ukhta State Technical University, 2016)

4. L. M. Ruzin, O. A. Morozyuk, S. M. Durkin, The effectiveness of methods to increase on the basis of laboratory and experimental studies Oil and Gas Business, 6, 408-423 (2014)

5. L. K. Altunina, V. S. Ovsyannikova, Yu. Z. Guseva, L. I. Svarovskaya, L. A. Astafyeva, D. A. Filatov, Complex physico-chemical and microbiological method for increasing oil recovery in low-temperature layers of high-viscosity oil Ecological bulletin of Russia, 6 , 14-17 (2014) 
6. A. P. Kondakov, S. V. Gusev, T. M. Surnova, Experience of the use of physical and chemical methods of enhanced oil recovery of YuS2 layers of Surgutneftegas OJSC fields Oil Industry, 9, 47-49 (2013)

7. A. G. Zakharyan, R. M. Musin, M. Cimic, Analyzing the efficiency of chemical EOR methods for oil fields developing by Rosneft Oil Company Oil Industry, 8, 58-59 (2015)

8. S. S. Kolomiets, P. M. Kosianov, V. G. Krasnov, Unconventional drilling methods V International scientific and practical conference of students, graduate students and scientists "Experience, current problems and prospects for the development of the oil and gas industry" ed. Yu B Chebykina (Tyumen: Industrial University of Tyumen), 67$76(2015)$

9. A. V. Temerko, Pat. 2208141 Russian Federation, E21B43/24. Method of development of oil and gas condensate fields. Patentee Temerko Alexandr. No: 2002128698/03; declare 28/10/2002; publ. 10/07/2003, Bull. No. 19.

10. P. M. Kosianov, V. G. Krasnov, Quantitative estimation of optimal parameters for drilling tools VIII International scientific and practical conference of students, graduate students and scientists "Experience, current problems and prospects for the development of the oil and gas industry" ed. Yu B Chebykina, 66-71 (Tyumen: Industrial University of Tyumen, 2018)

11. A. T. Khudaiberdiev, On the prospects for the use of chemical methods of oil recovery Scientific and practical conference "Actual problems of the petrochemical industry", 9799 (Moscow: VNIIPnef, t2019)

12. V. G. Krasnov, A. V. Krever, P. M. Kosianov, Pat. 155161 Russian Federation, IPC: E21B7/14, E21C37/16. Drilling tools. Patentee Victor Krasnov. No: 2015112010/03; declare 04/02/2015; publ. 09/27/2015, Bull. No. 27.

13. P. M. Kosianov, V. G. Krasnov, Optimization of the parameters of a drilling tool to improve its efficiency Nedropolzovaniye XXI vek (Subsoil use. XXI century), 3, 62-66 (2019)

14. A. T. Khudaiberdiev, Ways to improve the efficiency of oil production. Problems and prospects of their application IX International scientific and practical conference of students, graduate students and scientists "Experience, current problems and prospects for the development of the oil and gas industry" ed. Yu B Chebykina, $167-171$ (Tyumen: Industrial University of Tyumen, 2019)

15. P. M. Kosianov, Ways to Improve Production Efficiency. Problems and Ways of Their Solution Journal of Computational and Theoretical Nanoscience, 16, 3094-3097 (2019)

16. V. M. Dobrynin, B. Y. Wendelstein, D. A. Kozhevnikov, Petrophysics (Rock Physics). Moscow, Oil and Gas, 367 (2004) 